\title{
1.13 HYPONATREMIA
}

Hyponatremia, defined as a serum sodium value less than $135 \mathrm{mEq} / \mathrm{L}$, is the most common electrolyte disorder observed in hospitalized patients in the United States, occurring in up to $60 \%$ of patients. ${ }^{1}$ The disorder may develop within 48 hours of, or during, hospitalization (acute), or may be subacute or chronic. When it develops in the hospital, hyponatremia is associated with increased length of stay, increased cost of hospitalization, increased in-hospital mortality, and increased postdischarge mortality. Even chronic hyponatremia present at hospital admission adversely affects outcomes-such patients have a 30\% higher risk of mortality and are hospitalized 14\% longer than patients without hyponatremia at admission. ${ }^{2,3}$ Hospitalists can facilitate the evaluation and management of hyponatremia to improve patient outcomes, as well as decrease healthcare costs and length of stay.

\section{KNOWLEDGE}

Hospitalists should be able to:

- Distinguish acute from chronic hyponatremia.

- Identify hospitalized patients at risk of developing hyponatremia and institute monitoring measures to increase early recognition.

- Describe the symptoms of mild and severe hyponatremia.

- Describe the indicated serum and urine laboratory tests used to evaluate the causes of hyponatremia.

- Differentiate among hypertonic, isotonic, and hypotonic forms of hyponatremia.

- Identify the likely pathophysiologic process underlying a patient's hyponatremia on the basis of urine osmolality and electrolyte concentrations.

- Identify the likely pathophysiologic process underlying a patient's hyponatremia on the basis of the clinical volume status and urine sodium value.

- Explain how concurrent fluid administration or diuresis may affect urinary tests used in the evaluation of hyponatremia.

- Explain the physiology leading to development of the syndrome of inappropriate antidiuretic hormone secretion (SIADH) and describe how it is diagnosed.

- Recognize indications for specialty consultation, such as endocrinology or nephrology.

- Describe an appropriate treatment strategy for patients with asymptomatic, mildly symptomatic, and severely symptomatic hyponatremia, including the risks of treatment.

- Explain the appropriate rate of correction for acute or chronic hyponatremia, adjusted to the needs of the individual patient.

- Explain the indications for water restriction in hyponatremia.

- Explain the indications of isotonic sodium chloride fluid administration in hyponatremia.
- Explain the indications for hypertonic sodium chloride fluid administration in hyponatremia.

- Explain the role, limitations, risks, and contraindications of vasopressin receptor agonists in the treatment of hyponatremia.

- Predict how concurrent correction of other electrolyte disorders (eg, hypokalemia) may affect sodium correction.

\section{SKILLS}

Hospitalists should be able to:

- Elicit a thorough and relevant medical history, perform a physical examination, and review the medical record for factors contributing to the development of hyponatremia.

- Accurately assess the relevant volume status and neurologic examination findings of a patient with hyponatremia.

- Order and interpret indicated diagnostic studies that may include serum electrolytes, serum and urine osmolality, serum blood urea nitrogen, creatinine, uric acid, urine sodium, thyrotropin, and early-morning cortisol.

- Formulate and implement the most appropriate intervention tailored to the individual patient's etiology of hyponatremia while minimizing potential complications from overcorrection or undercorrection.

- Identify the most appropriate care setting to monitor patients with hyponatremia, including indications to transfer to the intensive care unit.

- Recognize symptoms and signs of severe hyponatremia and osmotic demyelination syndrome.

- Communicate with patients and families to explain the significance, etiology, and importance of recognizing and treating hyponatremia.

- Communicate with patients and families to explain the risks, monitoring, and appropriate management of hyponatremia.

- Document the treatment plan and provide clear discharge instructions for postdischarge clinicians.

- Facilitate coordination of transitional monitoring of recurrent hyponatremia after hospital discharge.

\section{ATTITUDES}

Hospitalists should be able to:

- Follow evidence-based recommendations when managing hospitalized patients with hyponatremia.

- Acknowledge the opportunity to decrease mortality, length of stay, and healthcare costs by addressing hyponatremia, even when asymptomatic.

\section{SYSTEM ORGANIZATION AND IMPROVEMENT}

To improve efficiency and quality within their organizations, hospitalists should:

- Lead, coordinate, and/or participate in multidisciplinary initiatives to promote patient safety and optimize management strategies for hyponatremia. 
- Participate in initiatives to articulate, manage, or restrict the use of high-risk therapies, such as hypertonic saline.

\section{References}

1. Upadhyay A, Jaber BL, Madias NE. Epidemiology of hyponatremia. Semin Nephrol. 2009;29(3):227-238

2. Nagler EV, Vanmassenhove J, van der Veer SN, Nistor I, Van Biesen W, Webster AC, Vanholder R. Diagnosis and treatment of hyponatremia: a systematic review of clinical practice guidelines and consensus statements. BMC Medicine. 2014;12:1.

3. Wald R, Jaber BL, Price LL, Upadhyay A, Madias NE. Impact of hospital-associated hyponatremia on selected outcomes. Arch Intern Med. 2010;170(3):294-302. 